\title{
Riqueza y abundancia de aves en bosques ribereños de diferentes anchos en la microcuenca del río Sesesmiles, Copán, Honduras
}

\author{
Inty T. Arcos, Francisco Jiménez, Célia A. Harvey \& Fernando Casanoves \\ CATIE, Turrialba, Costa Rica. Tel: 5582651. Fax: 5562598; iarcos@catie.ac.cr; fjimenez@catie.ac.cr; charvey@catie. \\ ac.cr; casonoves@catie.ac.cr
}

Recibido 22-IV-2006. Corregido 23-IV-2007. Aceptado 27-VIII-2007.

\begin{abstract}
Richness and abundance of birds in riparian forest belts of varied breadths at the Sesesmiles river microwatershed, Copan, Honduras. Riparian forests protect many species of plants and animals. We studied bird communities in riparian forest belts of the Sesesmiles river microwatershed, Copan, Honduras $\left(140^{\circ} 43^{\prime} 12^{\prime \prime}-140^{\circ} 58^{\prime} 35^{\prime \prime} \mathrm{N}, 88^{\circ} 53^{\prime} 23^{\prime \prime}-89^{\circ} 14^{\prime} 17^{\prime} \mathrm{W}\right)$. The main goal was to explore the effects of belt breadth on the richness and abundance of avian species visiting these forests. We selected 20 belts, and randomly established 30 observation points to monitor bird presence in the dry (March-April 2005) and rainy (June-July 2005) season ( $\mathrm{N}=60$ observations). A total of 1294 birds belonging to 145 species were recorded. Bird diversity was significantly correlated to the breadth of the riparian belts, with a greater number of species and individuals in belts $50 \mathrm{~m}$ wide or wider. Insectivorous and nectarivorous birds were the most abundant guilds. All bird species identified depend to some degree on riparian forests and are affected by belt breadth. Riparian belts over $50 \mathrm{~m}$ should be kept or established in order to conserve bird populations within agricultural and fragmented landscapes in similar tropical areas. Rev. Biol. Trop. 56 (1): 355-369. Epub 2008 March 31.
\end{abstract}

Key words: avian biodiversity, forest dependence, riparian forests, trophic levels.

Los ecosistemas ribereños albergan gran diversidad de hábitats que benefician a un alto número de especies de plantas y animales (Kauffman y Kruger 1984). Dentro de la selva tropical los bosques ribereños son apenas evidentes, pero en áreas abiertas donde la agricultura y ganadería son los principales usos de la tierra. Los bosques ribereños son pequeños parches de vegetación que quedan en los paisajes alterados, formando galerías evidentes que funcionan como corredores biológicos, y a veces como único hábitat para la fauna silvestre (Nores et al. 2005). Varios estudios realizados, principalmente en zonas templadas, han documentado la importancia de los bosques ribereños para las poblaciones de aves (Skagen et al.1998), notando que la composición poblacional de las aves en estos ecosistemas es de mayor riqueza y abundancia que en las áreas vecinas a los bosques ribereños (Lock y Naiman 1998, Skagen et al.1998). Por ejemplo, estudios realizados en Montana, Estados Unidos, indican que el 59\% de las especies de aves usan los bosques ribereños para reproducción, refugio y como lugar de alimentación (McLean et al. 1963, Skovlin 1967, Paulsen 1969).

Estudios recientes sugieren que las comunidades de aves de los ecosistemas ribereños pueden ser afectadas por la deforestación y modificación ambiental de los paisajes vecinos. Smith y Johnstone (1977), y Woinarski et al. (2000) encontraron una reducción de las especies de aves consideradas como especies ribereñas en Australia, asociada con el deterioro de la vegetación ribereña. El ancho de los bosques ribereños puede ser un factor importante en los ensamblajes de las comunidades de aves presentes en estos ecosistemas.

La microcuenca del río Sesesmiles en Copán, Honduras se caracteriza por ser una 
zona agrícola, donde predominan los cultivos de maíz, café y áreas de ganadería. Las franjas ribereñas que aún existen, se localizan en la parte alta de la microcuenca; generalmente son áreas pequeñas, aisladas y de anchos diferentes. Estos bosques son componentes importantes del paisaje, y en varios casos, son los únicos remanentes de bosque en la microcuenca. Existen pocas investigaciones en los trópicos, sobre los ecosistemas ribereños, su valor de conservación, condición actual o habilidad de soportar la presión de uso (Sattler 1993 y Douglas y Pouliot 1997 citados en Woinarski et al. 2000), así como su papel como hábitat para las aves. Los objetivos del estudio fueron caracterizar la riqueza y la abundancia de aves presentes en los bosques ribereños y explorar el efecto del ancho de franja de la vegetación ribereña sobre las comunidades de aves presentes, con el fin de tener información para apoyar la toma de decisiones en las políticas de conservación de estos ecosistemas.

\section{MATERIALES Y MÉTODOS}

Descripción del sitio de estudio: La microcuenca del río Sesesmiles mide $38 \mathrm{~km}^{2}$, y se localiza al occidente de Honduras, en el departamento de Copán (140 43' 12" - 140 $58^{\prime} 35^{\prime \prime} \mathrm{N}$, y $88^{\circ}$ 53' 23" - 89 $14^{\circ}$ ' 17' W). Forma parte de la subcuenca del río Copán, cuenca del río Motagua que sirve de línea fronteriza entre Honduras y Guatemala. La precipitación promedio anual es de $1609 \mathrm{~mm}$, con un ámbito de 1425 a $1760 \mathrm{~mm}$. El mes más lluvioso es septiembre (promedio de $229 \mathrm{~mm}$ ) y el menos lluvioso es marzo (promedio de $11 \mathrm{~mm}$ ); el periodo seco dura cinco meses (diciembre a abril). La topografía de la microcuenca es bastante quebrada, con fuertes pendientes y pocas zonas planas, ubicadas en áreas cercanas a los cauces de los ríos.

La altitud varía de 600 a $1600 \mathrm{msnm}$. Las temperaturas mínimas y máximas promedian 16 y $26^{\circ} \mathrm{C}$, respectivamente. Los bosques de la microcuenca pertenecen a la zona de vida del bosque tropical seco. Son bosques latifoliados a semideciuos, con características de bosques nublados a húmedos en las zonas más altas. Los usos actuales del suelo predominantes en la microcuenca de río Sesesmiles son cafetales $(10 \%)$, pasturas $(5 \%)$ y cultivos anuales $(11 \%)$. En general los bosques ribereños se ubican en la parte media y alta de la microcuenca. Son bosques secundarios, sin especies maderables y con un alto nivel de perturbación por la ganadería que utiliza las quebradas como abrevaderos y por la agricultura tradicional de tumba y quema.

Selección de los sitios de estudio: Se recorrió el área de estudio con el propósito de localizar todos los parches de bosque ribereño ubicados en la parte media y alta de la microcuenca del río Sesesmiles. En el campo se localizó y se determinó mediante un sistema de posicionamiento global, preliminarmente una muestra de 25 bosques ribereños distintos, de manera aleatoria, en donde se realizó el muestreo de aves. Se identificaron únicamente bosques que se encontraban rodeados por pastizales y monocultivos de maíz, café o fríjol. Otro criterio de selección fue dado por el orden de bifurcación de los cauces dentro de la red de drenaje natural de la microcuenca. Fueron seleccionados los bosques que se encontraban en las márgenes de los cauces de orden uno (cauces que no cuentan con ningún tributario) (Villón 2002). Como una herramienta para facilitar la ubicación de los cauces, se utilizó un plano de la microcuenca que incluía la red de drenaje natural.

Muestreo de anchos de franjas ribereñas: En cada uno de los 25 bosques que cumplían con los criterios de selección antes mencionados, se realizaron seis mediciones de anchos de franjas ribereñas, tomando como punto de referencia las desembocaduras de los cauces de orden uno en los cauces de orden dos, según la red de drenaje. A partir del punto de convergencia de los cauces, se midieron $100 \mathrm{~m}$ a lo largo del cauce de orden uno, donde se estableció el primer punto 
de medición de ancho de franja; los siguientes puntos de mediciones fueron separados cada 100 m (Fig. 1).

Con los datos de anchos de franja de los 25 bosques medidos, se elaboró una tabla de frecuencias de los diferentes anchos. De acuerdo a esta distribución se seleccionaron 20 bosques ribereños y 60 anchos de franja de los bosques seleccionados a priori. En cada uno de estos anchos de franja ribereña se estableció un punto de conteo de aves. Las 60 franjas fueron agrupados en tres categorías (hasta $10 \mathrm{~m}, \mathrm{n}=20$, hasta $50 \mathrm{~m}, \mathrm{n}=28$ y más de $50 \mathrm{~m}$ de ancho, $\mathrm{n}=12$ ), las cuales se utilizaron como variables clasificatorias para el análisis de varianza.

Metodología para el monitoreo de las comunidades de aves: Para caracterizar las comunidades de aves en los bosques ribereños se utilizó el método de conteos por puntos, que es el principal procedimiento de monitoreo de aves terrestres debido a su eficacia en todo tipo de terreno y hábitat (Ralph 1995 y Ralph et al 1996). En los 20 bosques ribereños seleccionados se establecieron al azar 30 puntos de observación de individuos y especies de aves. Se realizó un muestreo temporal, uno en la época seca (marzo - abril del 2005) y otro en la época lluviosa (junio-julio del 2005), para un total de 60 observaciones. Cada punto de conteo estuvo separado entre si por una distancia mínima de $100 \mathrm{~m}$ uno del otro. Con el fin de disminuir la probabilidad de registrar el mismo individuo en los diferentes puntos de observación donde se realizaron los conteos (Ralph 1995 y Ralph et al. 1996), el observador se ubicó junto al cauce del río. Alrededor de cada uno de los puntos de conteo de aves ubicados a lo largo del cauce, se fijó un radio de observación de $25 \mathrm{~m}$. En los bosques cuyo ancho fue inferior a $25 \mathrm{~m}$, se tomaron en cuenta sólo las aves que estaban utilizando la vegetación ribereña y no

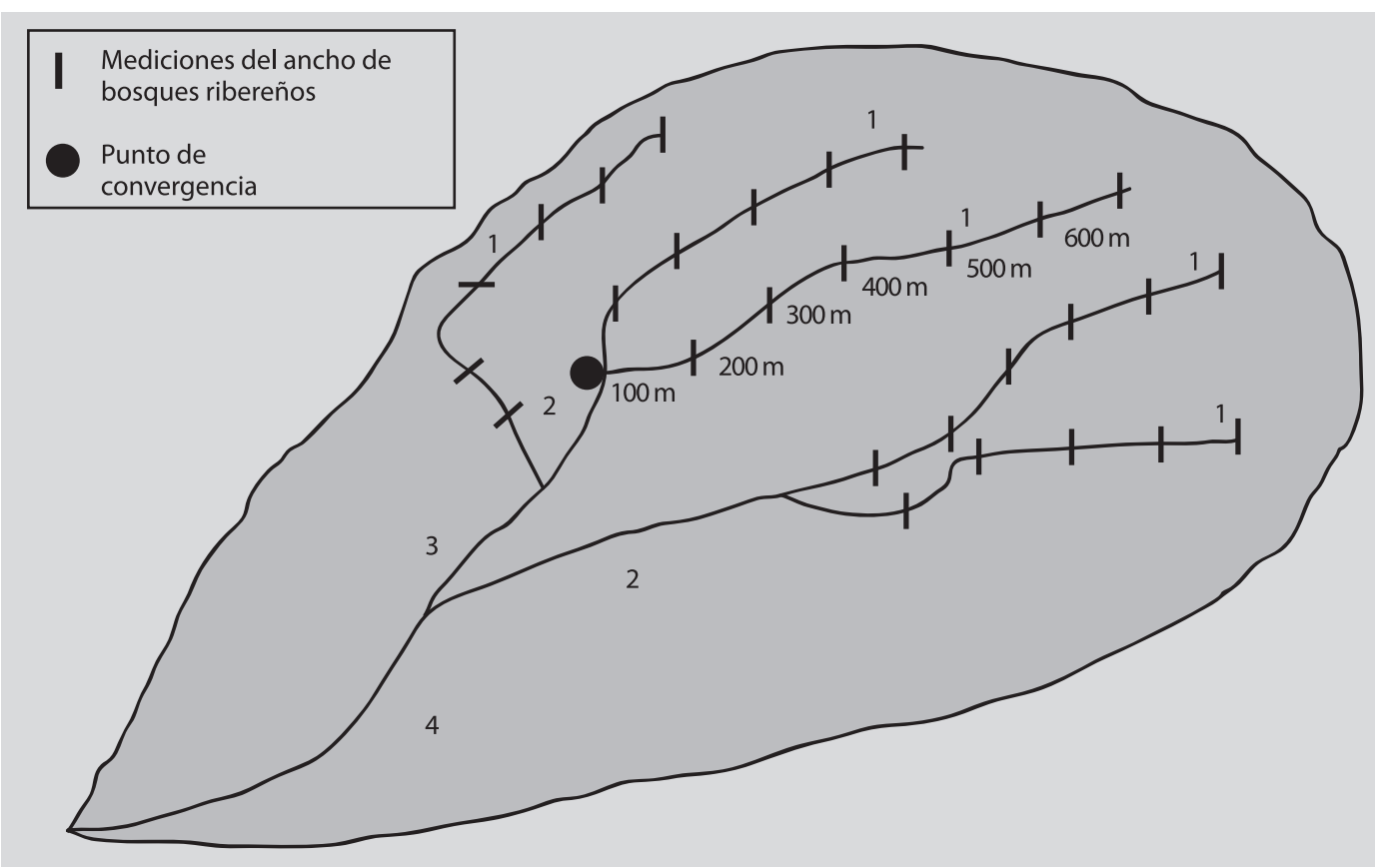

Fig. 1. Numeración del orden de la red de drenaje y puntos de medición del ancho de la franja de los bosques ribereños, en los cauces de orden uno de la microcuenca del río Sesesmiles, Copán, Honduras, 2005.

Fig. 1. Drainage net numeration order and measurement sites of riparian forest breadth in first order river channels of the Sesesmiles river microwatershed, Copan, Honduras, 2005. 
las que se observaron en las áreas de cultivo o pastizales, siguiendo la metodología propuesta por Lock y Naiman (1998).

Se realizaron las observaciones para identificar visual y auditivamente todas las especies que se encontraban en un ámbito limitado de $25 \mathrm{~m}$ de radio (Ralph et al. 1996), apoyándose en el uso de binoculares y guías de campo. Se accedió al lugar de conteo causando el mínimo disturbio sobre las aves. Una vez que se llegó al punto de conteo, se dio un periodo de espera de 5 min antes de iniciar el mismo, a fin de minimizar el efecto que pueda tener el observador sobre el comportamiento de las aves (Villarreal et al. 2004). El observador permaneció por un periodo de $15 \mathrm{~min}$ en cada punto de conteo de aves, y las observaciones fueron realizadas entre las 6 y 9 de la mañana.

Análisis estadístico de los datos: Se combinaron los datos, tanto de especies como de individuos, de las aves observadas en la época lluviosa y seca, y se calculó el número de individuos y especies de aves, para cada punto en donde previamente se había tomado el ancho real de la franja de los bosques ribereños. Para cada uno de los puntos se calcularon los índices de diversidad de Shannon, Margalef y Simpson. También se clasificaron las especies encontradas por familia y gremio alimentario: frugívoro, insectívoro, granívoro, nectarívoro y carnívoro, utilizando la clasificación de Stiles (1985). Con cada uno de los gremios alimentarios se realizó un análisis de varianza del número de especies e individuos para probar la hipótesis sobre el efecto de los diferentes anchos de franjas ribereñas.

También se clasificaron las especies de aves según el grado de dependencia del bosque ribereño, siguiendo la metodología propuesta por Stiles (1985), de la siguiente forma: $1=$ obligadas $1-2$ a $3=$ dependientes y $2-3$ a $3=$ independientes, a fin de detectar las diferencias en la cantidad de aves en los diferentes anchos de franjas ribereñas. Con los resultados de la aplicación de los índices de diversidad y las posteriores reclasificaciones de las espe- cies, se realizó un análisis de varianza con el programa InfoStat (InfoStat 2004), utilizando las categorías de anchos como las variables de clasificación, y la cobertura de los estratos (\%), diámetro a la altura del pecho (DAP) y la altitud (msnm del bosque ribereño) como covariables. Además se comparó la riqueza de especies observadas y las especies esperadas, utilizando los estimadores de Chao 1 y Ace, para los diferentes anchos de franja ribereña, y se determinó el porcentaje de especies esperadas para los diferentes anchos de franjas ribereñas muestreadas.

\section{RESULTADOS}

Caracterización vegetal y estructural en cada punto de observación de aves en los diferentes anchos de franja ribereña: Las franjas ribereñas con anchos superiores a $50 \mathrm{~m}$ presentaron mayor cobertura de la vegetación en cada estrato, sin embargo, la variabilidad estructural fue mayor en las franjas ribereñas con anchos menores de $10 \mathrm{~m}$ (Cuadro 1). Además se encontró que el promedio del DAP de los árboles mayores de $10 \mathrm{~cm}$ de diámetro, fue superior en las franjas mayores que $50 \mathrm{~m}$ en comparación a los demás anchos evaluados $(\mathrm{F}=5.30, \mathrm{gl}=2, \mathrm{p}=0.0077)$. Los promedios de DAP encontrados en franjas mayores a $50 \mathrm{~m}$, entre 50 y $10 \mathrm{~m}$ y menores que $10 \mathrm{~m}$ fueron, respectivamente: $65.3,36.1$ y $34.2 \mathrm{~cm}$.

Distribución de las especies y gremios alimentarios de aves en la microcuenca del río Sesesmiles: En los 60 puntos de observación distribuidos en los 20 bosques ribereños de la microcuenca en estudio, se encontraron 1 294 aves de 145 especies. Las especies encontradas pertenecen a 34 familias, de las cuales la familia Icteridae fue el grupo con mayor número de especies registradas, con un $22 \%$, seguido por la familia Turdidae con un $12 \%$ del total de especies encontradas en el estudio. Entre las especies más comunes encontradas en los diferentes anchos de franjas ribereñas 


\section{CUADRO 1}

Valores promedio de la variabilidad estructural del bosque ribereño en los diferentes puntos de observación de aves, en la microcuenca del río Sesesmiles, Copán, Honduras, 2005

TABLE 1

Average values of the structural characteristics of riparian forests in sites where birds were observed in the Sesesmiles river microwatershed, Copan, Honduras, 2005

Franjas de bosque ribereño No. de puntos de muestreo Índice de cobertura del follaje Variabilidad estructural

$\begin{array}{cccc}<10 \mathrm{~m} & 20 & 3.25 \pm 0.43 \mathrm{a}^{*} & 59.79 \\ 10-50 \mathrm{~m} & 28 & 5.82 \pm 0.64 \mathrm{~b} & 58.03 \\ >50 \mathrm{~m} & 12 & 9.67 \pm 1.21 \mathrm{c} & 43.52\end{array}$

*Letras distintas indican diferencias significativas ( $\mathrm{p}<=0.05$ ), entre franjas de bosque de diferentes anchos.

*Different letters indicate significant statistical differences $(\mathrm{p}<=0.05)$, among different forest breadths.

están: Zonotrichia capensis septentrionales, Xenotriccus callizonus, Zenaida asiatica, y Turdus grayi (Cuadro 2).

Se observaron 24 especies migratorias que corresponden al 17\% del total de especies observadas dentro de las franjas ribereñas. Las especies migratorias con más individuos registrados fueron: Wilsonia pusilla (16 idividuos), Vireo pinicolus (13 individuos), Myiodynastes luteiventris (18 individuos), Icterus galbula (9 individuos) y Dendroica virens (8 individuos). En total se registraron 417 individuos de especies migratorias, que corresponden al 32\% del total de individuos registrados. Se identificaron seis gremios alimentarios; el gremio con mayor número de especies asociadas fue los insectívoros con el $43 \%$ del total de especies y $33 \%$ del total de individuos, seguido por el grupo de los omnívoros con $29 \%$ del total de especies y $40 \%$ del total de individuos (Cuadro 3).

\section{CUADRO 2}

Diez de las especies más comunes encontradas en los distintos anchos de bosques ribereños. Copán, Honduras, 2005

TABLE 2

Ten of the most common species found in the riparian forests of the Sesesmiles river microwatershed, Copan, Honduras, 2005

\section{Anchos de franjas ribereñas}

\section{$0-10 \mathrm{~m}$}

Turdus grayi

Saltator atriceps

Quiscalus mexicanus

Dives dives

Centurus aurifrons

Cyanocorax morio

Momotus momota

Psarocolius montezuma

Icterus pectorales

Contopus cinereus

$$
\text { 10-50 m }
$$

Zonotrichia capensis septentrionales

Xenotriccus callizonus

Zenaida asiatica

Wilsonia pusilla

Xiphocolaptes promeropirhynchus

Volatinia jacarina

Wilsonia citrina

Vireo pinicolus

Dendroica graciae

Sittasomus griseicapillus
$>50 \mathrm{~m}$

Zenaida asiatica

Xenotriccus callizonus

Glaucidium brasilianum

Myiodynastes luteiventris

Oncostoma cinereigulare

Laterallus ruber

Xiphocolaptes promeropirhynchus

Herpetotheres cachinnans

Passerina cyanea

Melanerpes formicivorus 
CUADRO 3

Gremios alimenticios en la población de aves identificadas en los bosques ribereños en la microcuenca del río Sesesmiles, Copán, Honduras, 2005

TABLE 3

Feeding guilds of birds identified in riparian forests of the Sesesmiles river microwatershed, Copan, Honduras, 2005

$\begin{array}{lcccc}\text { Gremio alimenticio } & \text { No. de especies } & \begin{array}{c}\text { Proporción total } \\ \text { de especies (\%) }\end{array} & \text { No. de individuos } & \begin{array}{c}\text { Proporción } \\ \text { de individuo }\end{array} \\ \text { Insectívoros } & 63 & 43 & 439 & 33 \\ \text { Omnívoros } & 42 & 29 & 524 & 40 \\ \text { Granívoros } & 14 & 10 & 87 & 7 \\ \text { Frugívoros } & 12 & 8 & 164 & 13 \\ \text { Nectarívoros } & 8 & 6 & 55 & 4 \\ \text { Carnívoros } & 6 & 3 & 25 & 3 \\ \text { Total } & 145 & 100 & 1294 & 100\end{array}$

En los bosques ribereños con franjas de 0 a $10 \mathrm{~m}$ de ancho se encontraron 14 especies, en franjas con anchos de 10 a $50 \mathrm{~m}$ se encontraron 22 especies y en franjas con anchos mayores a $50 \mathrm{~m}$ se encontraron 28 especies. Los tres diferentes ámbitos de anchos de franja ribereña compartieron un $30 \%$ del total de especies registradas en el estudio.

\section{Análisis comparativo de la población de} aves con respecto a las categorías de anchos de franjas ribereñas: Se encontró diferencias en el número de especies observadas y de especies esperadas (estimadores de Chao 1 y ACE) para los diferentes anchos de franja (Cuadro 4).
La curva de acumulación al nivel de paisaje, que relaciona el número de especies con el número de individuos (Fig. 2), aplicando el índice de Chao 1, permitió estimar que se muestreó el $87 \%$ de las especies esperadas del todo el paisaje de la microcuenca del río Sesesmiles, con una alta representatividad de especies e individuos en los diferentes bosques ribereños evaluados.

Hubo diferencias significativas en el número de especies $(\mathrm{F}=13.84, \mathrm{gl}=2, \mathrm{p}=0.0001)$ $\mathrm{y}$ en el número de individuos de aves $(\mathrm{F}=12.51$, $\mathrm{gl}=2, \mathrm{p}=0.0001$ ) entre los diferentes anchos de franja de los ecosistemas ribereños. Al aumentar el ancho de franja ribereña, se incrementó la

CUADRO 4

Estimación de la riqueza total de especies de aves presentes en los diferentes anchos de franjas ribereñas de la microcuenca del río Sesesmiles, Copán, Honduras, 2005

TABLE 4

Estimation of total bird richness in different riparian forests of different breadths in the microwatershed of the Sesesmiles river, Copan, Honduras, 2005

\begin{tabular}{|c|c|c|c|c|c|}
\hline \multirow{2}{*}{$\begin{array}{l}\text { Anchos de franjas } \\
\text { ribereñas }\end{array}$} & \multirow{2}{*}{$\begin{array}{l}\text { Especies } \\
\text { observadas }\end{array}$} & \multicolumn{2}{|c|}{ Especies esperadas } & \multirow{2}{*}{$\begin{array}{c}\text { Promedio de especies } \\
\text { esperadas }\end{array}$} & \multirow{2}{*}{$\begin{array}{c}\text { Eficiencia de muestreo } \\
(\%)\end{array}$} \\
\hline & & Chao 1 & Ace & & \\
\hline $0-10 \mathrm{~m}$ & 71 & 96.0 & 93.0 & 95 & 75 \\
\hline $10-50 \mathrm{~m}$ & 95 & 113.0 & 120.0 & 117 & 81 \\
\hline$>50 \mathrm{~m}$ & 98 & 112.4 & 111.1 & 223 & 44 \\
\hline
\end{tabular}




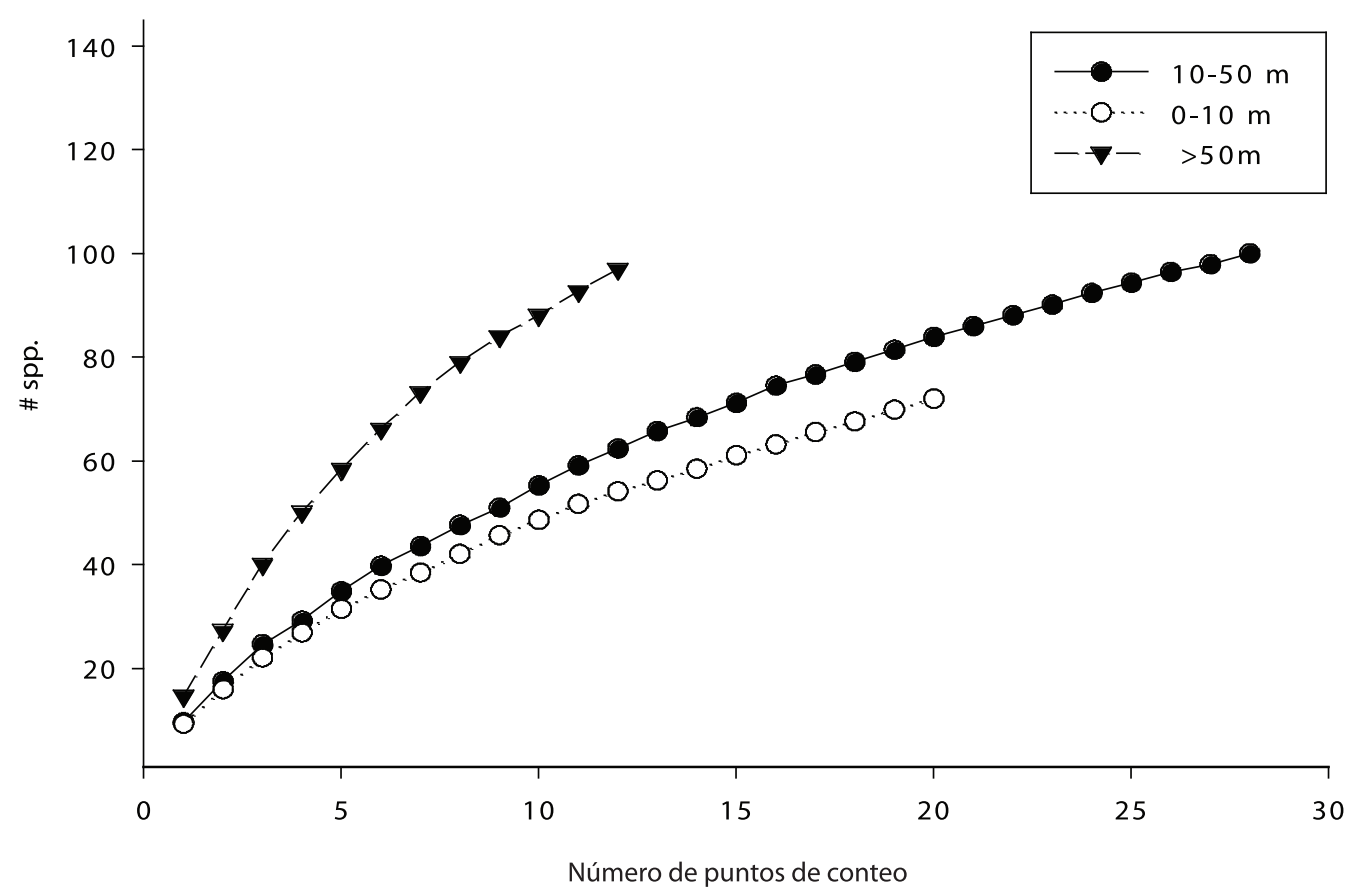

Fig. 2. Curvas de acumulación de especies en franjas de bosques ribereños con diferentes anchos, en la microcuenca de río Sesesmiles, Copán, Honduras, 2005.

Fig. 2. Species accumulation curves in different riparian forests of different breadths in the Sesesmiles river microwatershed, Copán, Honduras, 2005.

cantidad de especies e individuos encontrados: en franjas mayores de $50 \mathrm{~m}$ fue mayor el número de especies e individuos en comparación con las franjas menores de $50 \mathrm{~m}$ y menores de $10 \mathrm{~m}$. (Fig. 3). En el análisis comparativo entre los individuos de las diferentes especies se encontró que la especie Turdus grayi fue la más abundante con $9 \%$ de la población, seguida por Dives dives con 7\% y Saltator atriceps con $6 \%$.

También se encontraron diferencias en los índices de diversidad de Shannon y Margalef, entre los diferentes anchos de franjas ribereñas. Las franjas mayores a $50 \mathrm{~m}$ presentaron mayores valores de riqueza y abundancia de especies de aves, con relación a los anchos menores. Para el índice de Simpson no hubo diferencias significativas entre las categorías de anchos de franja (Cuadro 5).

Hubo diferencias en la composición de especies presentes en bosques de diferentes anchos; en las franjas mayores de $50 \mathrm{~m}$ se identificaron algunas especies que no se encontraron en franjas de anchos menores, como por ejemplo Catharus aurantiirostris y Trogon collares que son especies cuya dieta depende, en gran medida, de los árboles que producen frutas, los cuales se encuentran principalmente en bosques ribereños que han sufrido menor deforestación y que conservan franjas anchas. Lo mismo ocurrió con la especie Laterallus ruber, la cual está relacionada con ambientes húmedos y se alimenta de insectos acuáticos, peces pequeños, ranas y otras especies relacionadas al cauce del río (Howell y Webbs 1995). Estas especies podrían ser indicadoras de la salud del bosque ribereño, y su hábitat parece estar estrechamente relacionado a las franjas ribereñas.

Los grupos alimenticios insectívoros y nectarívoros también presentaron diferencias significativas entre franjas de bosques ribereños 


\section{CUADRO 5}

Índices de diversidad de aves y su relación con los anchos de franjas ribereñas en la Microcuenca del Río Sesesmiles,

Copán, Honduras, 2005

TABLE 5

Bird diversity indices and their relation to riparian forest breadth in the Sesesmiles River Microwatershed, Copan, Honduras, 2005

\begin{tabular}{|c|c|c|c|c|c|}
\hline \multirow[b]{2}{*}{ Índices } & \multirow[b]{2}{*}{ Valor F } & \multirow[b]{2}{*}{ Probabilidad } & \multicolumn{3}{|c|}{ Promedios de los anchos franjas ribereña } \\
\hline & & & $\begin{array}{c}<10 \mathrm{~m} \\
\mathrm{n}=20\end{array}$ & $\begin{array}{c}10-50 \mathrm{~m} \\
\mathrm{n}=28\end{array}$ & $\begin{array}{c}>50 \mathrm{~m} \\
\mathrm{~N}=12\end{array}$ \\
\hline Margalef & 10.31 & 0.0002 & $3.02 \mathrm{~b}$ & $2.96 \mathrm{~b}$ & $4.13 \mathrm{a}^{*}$ \\
\hline Shannon & 8.49 & 0.0006 & $2.09 \mathrm{~b}$ & $2.10 \mathrm{~b}$ & $2.49 \mathrm{a}$ \\
\hline Simpson & 2.11 & 0.1305 & $0.08 \mathrm{a}$ & $0.10 \mathrm{a}$ & $0.07 \mathrm{a}$ \\
\hline
\end{tabular}

*Letras distintas indican diferencias significativas $(\mathrm{p}<0.05)$, entre franjas de diferentes anchos.

*Different letters indicate significant statistical differences $(\mathrm{p}<0.05)$, among different riparian forests of different breadths.

de diferentes anchos (Cuadro 6). Se encontró mayor número de individuos y especies de aves insectívoros y nectarívoros en las franjas mayores de $50 \mathrm{~m}$ que en franjas menores a 50 m. Los demás gremios alimenticios no presentaron diferencias significativas en el número de especies e individuos entre los diferentes anchos de franja ribereña.

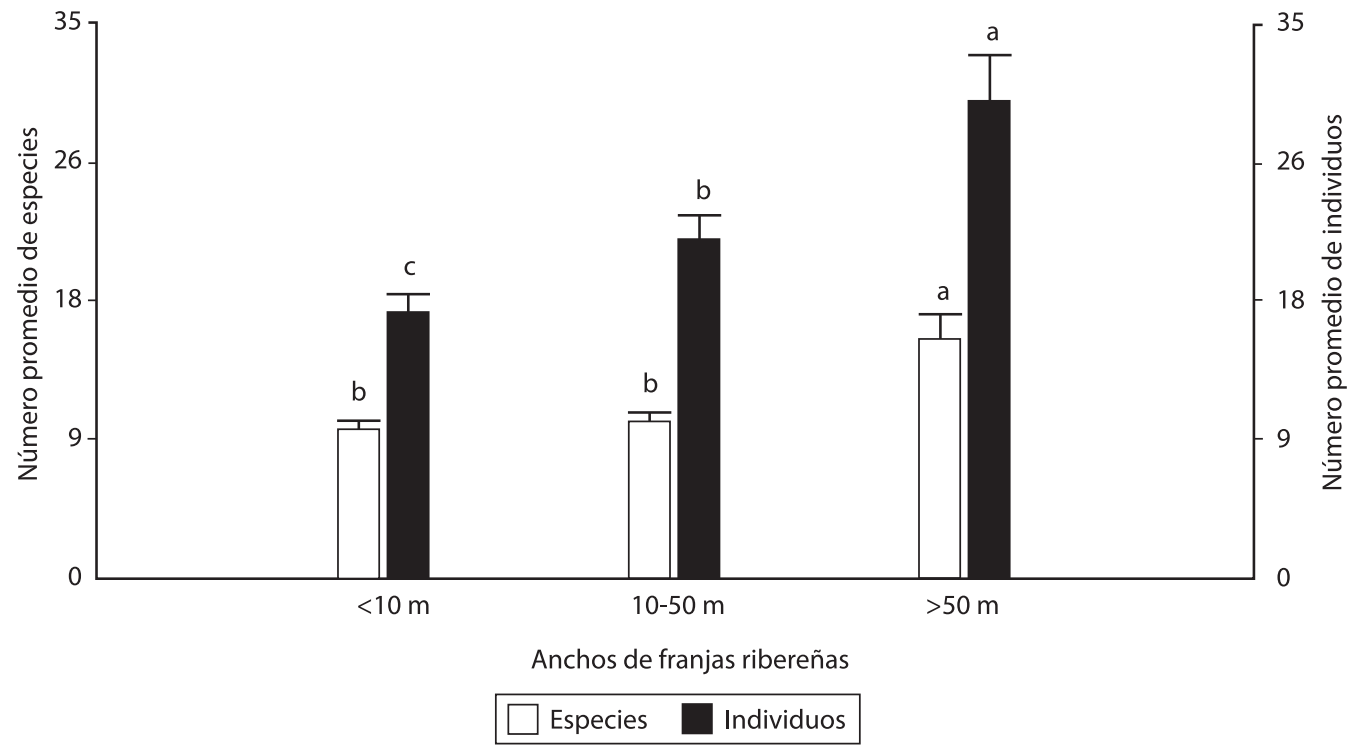

Fig. 3. Número promedio de especies e individuos observados por puntos de anchos de franjas ribereñas en la Microcuenca del río Sesesmiles. Letras diferentes indican diferencias en el número de especies o individuos entre los diferentes anchos de franjas.

Fig. 3. Mean number of species and individuals observed in riparian forests of different breadths in the Sesesmiles river microwatershed. Different letters indicate differences in the number of existing species or individuals among different forest breadths. 
CUADRO 6

Número promedio de individuos y especies de aves por gremio alimenticio observadas en los puntos de los diferentes anchos de franja ribereña, microcuenca del río Sesesmiles, Copán, Honduras, 2005

TABLE 6

Average number of individuals and species of birds per feeding guild observed in riparian forests of different breadths in the Sesesmiles River microwatershed, Copan, Honduras, 2005

\begin{tabular}{|c|c|c|c|c|c|c|}
\hline \multirow[t]{2}{*}{ Gremios alimenticios } & \multirow[t]{2}{*}{ Variables } & \multirow[t]{2}{*}{ Valor F } & \multirow[t]{2}{*}{ Probabilidad } & \multicolumn{3}{|c|}{ en los anchos de franjas ribereñas } \\
\hline & & & & $<10 \mathrm{~m}$ & $10-50 \mathrm{~m}$ & $>50 \mathrm{~m}$ \\
\hline Insectívoros & individuos & 24.7 & 0.0001 & $3.15 \mathrm{c}^{*}$ & $7.04 \mathrm{~b}$ & $11.42 \mathrm{a}$ \\
\hline Insectívoros & Especies & 10.8 & 0.0001 & $2.90 \mathrm{~b}$ & $3.93 \mathrm{~b}$ & $6.25 \mathrm{a}$ \\
\hline Nectarívoros & individuos & 1.5 & 0.0109 & $0.55 \mathrm{~b}$ & $0.46 \mathrm{~b}$ & $1.67 \mathrm{a}$ \\
\hline Nectarívoros & Especies & 2.9 & 0.0016 & $0.25 \mathrm{~b}$ & $0.39 \mathrm{~b}$ & $1.25 \mathrm{a}$ \\
\hline Granívoros & individuos & 1.8 & 0.1809 & $1.85 \mathrm{a}$ & $1.04 \mathrm{a}$ & $0.75 \mathrm{a}$ \\
\hline Granívoros & Especies & 1.5 & 0.2271 & $1.05 \mathrm{a}$ & $0.64 \mathrm{a}$ & $0.67 \mathrm{a}$ \\
\hline
\end{tabular}

*Letras distintas indican diferencias significativas $(\mathrm{p}<=0.05)$, entre franjas de diferentes anchos.

*Different letters indicate significant statistical differences $(\mathrm{p}<0.05)$, among different riparian forest band breadths.

Análisis comparativo de población de aves con respecto a la dependencia del bosque: Según la clasificación de Stiles (1985), el 6\% de los individuos y $13 \%$ de las especies son especies obligadas (totalmente dependientes del bosque), el $26 \%$ de los individuos y el $39 \%$ de las especies son especies dependientes del bosque y el $68 \%$ de los individuos y $48 \%$ de las especies son independientes de los bosques.

Hubo diferencias estadísticamente significativas para las especies obligadas $(\mathrm{F}=7.13, \mathrm{gl}=2$, $\mathrm{p}=0.0017$ ) en los diferentes anchos de franjas ribereñas. El mayor promedio (3.67) de especies obligadas se registraron en bosques mayores a $50 \mathrm{~m}$. Para las especies dependientes se encontraron diferencias $(\mathrm{F}=9.2, \mathrm{gl}=2, \mathrm{p}=0.0003)$ con respecto a los anchos de franja y el promedio más alto (9.23) de especies se encontró en los anchos mayores a $50 \mathrm{~m}$ (Fig. 4).

Hubieron mas especies independientes del bosque en bosques mayores a $50 \mathrm{~m}$ que en otros anchos $(\mathrm{F}=7.64, \mathrm{gl}=2, \mathrm{p}=0.0012)$. Con las especies independientes del bosque se encontraron diferencias significativas $(\mathrm{F}=10.01$, $\mathrm{gl}=1, \mathrm{p}=0.0025$ ) cuando se utilizó el DAP como covariable, resultando que a mayor DAP, mayor número de especies independientes. También se encontró que el número de individuos $(\mathrm{F}=14.53, \mathrm{gl}=1, \mathrm{p}=0.0003)$ y especies $(\mathrm{F}=13.33, \mathrm{gl}=1, \mathrm{p}=0.0006)$ de aves nectarívoros fueron afectados por la covariable DAP.

\section{DISCUSIÓN}

Caracterización estructural de la vegetación ribereña en cada punto de observación de aves: En los bosques ribereños de la zona alta de la microcuenca del río Sesesmiles se encontró que en las franjas mayores de $50 \mathrm{~m}$, la cobertura del follaje y los promedios de DAP son mayores que en franjas más pequeñas. Esto puede deberse a que dichas características están relacionadas a la madurez del bosque, y que las franjas de bosque ribereño más anchas han sufrido menos presión. Sin embargo, la 


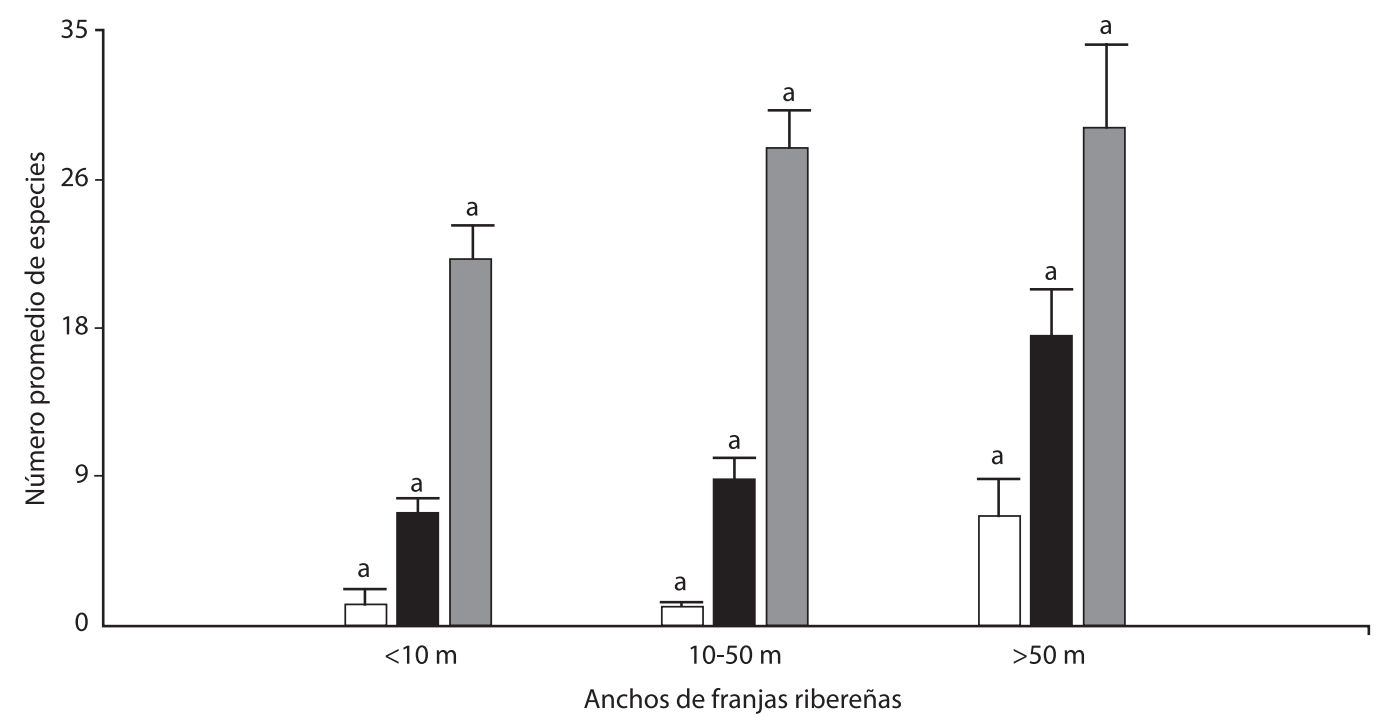

Especies

Individuos

Independientes

Fig. 4. Promedio de especies de aves encontradas en los tres niveles de dependencia hacia los bosques, en los diferentes anchos de franjas ribereñas. Letras diferentes indican diferencias entre los diferentes anchos de franjas ribereñas, microcuenca río Sesesmiles Copán, Honduras, 2005.

Fig. 4. Average number of species found in the three levels of dependence toward forests in the different riparian forest band breadths. Different letters indicate statistical differences in species average number among the three categories of dependence of bird species of birds toward the existing forests, Sesesmiles river microwatershed, Copan, Honduras, 2005.

variabilidad estructural fue mayor en las franjas ribereñas menores de $10 \mathrm{~m}$. La heterogeneidad de las franjas angostas puede estar relacionada a la mayor intervención antrópica en las mismas, la cual permite el desarrollo de especies pioneras e invasoras (Treviño et al. 2001).

Las características estructurales de la vegetación ribereña se encuentran estrechamente relacionadas con la diversidad y composición de la fauna del bosque. En el caso de las aves, la fragmentación del bosque afecta algunas especies, disminuyendo la disponibilidad de nichos para la alimentación y reproducción (Finegan et al. 2004). De esta manera, la vegetación de áreas ribereñas constituye un hábitat importante para comunidades de aves residentes y migratorias, las cuales se ven afectas por la disminución de la cobertura ribereña (Treviño et al. 2001).
Caracterización de la población de aves en los bosques ribereños en la microcuenca del río Sesesmiles: En los bosques ribereños, se registraron 1297 individuos pertenecientes a 145 especies, de las 715 registradas para Honduras, lo que representa el $20 \%$ de las especies de aves de este país, registradas en solamente $38 \mathrm{~km}^{2}$ del área de la microcuenca. Se registraron también en las franjas de bosque ribereño especies como Catharus aurantiirostris y Laterallus ruber, que son reportadas por Stiles y Skutch (1989) que son propias de este tipo de ecosistema. El elevado número de especies de aves registradas en las franjas ribereñas indica relevancia a estos ecosistemas para la conservación de la diversidad de aves, tanto a nivel nacional como regional, y apoya la toma de decisiones para incorporar y priorizar el manejo de los bosques ribereños en los planes de gestión de la microcuenca. 
El $17 \%$ de las especies y el $32 \%$ del total de individuos registrados dentro de las franjas ribereñas fueron especies migratorias, tales como Wilsonia pusilla, Vireo pinicolus, Myiodynastes luteiventris, Icterus galbula y Dendroica virens. Este porcentaje de especies migratorias encontrado en las franjas ribereñas de la microcuenca del río Sesesmiles es alto. En estudios realizados por Verea et al. (2000) en el norte de Venezuela, encontraron que las especies migratorias fueron más abundantes $(6.5 \%)$ en los bosques ribereños, mientras que solamente dos de las especies migratorias se encontraron en bosques continuos. Sin embargo, Klimas et al. (1981) reportan la existencia de 100 especies de aves que dependen o están asociadas a los ecosistemas ribereños del valle del Mississippi.

Número de individuos y especies encontrados en los diferentes anchos de franjas ribereñas: En los bosques ribereños de la microcuenca del río Sesesmiles se encontró que en franjas ribereñas mayores de $50 \mathrm{~m}$ se incrementan la cantidad de especies e individuos (Fig. 3). Estos datos concuerdan con lo hallado en Norteamérica por Stauffer y Best (1980) donde reportaron que la riqueza y abundancia en la población de aves se incrementa con el aumento del ancho de las franjas ribereñas. Spackman y Hughes (1995) en Vermont, Estados Unidos encontraron que franjas ribereñas con anchos iguales o mayores a $75 \mathrm{~m}$, son necesarias para la conservación de las poblaciones de aves. Shirley y Smith (2005), en un estudio realizado en Canadá, reportaron un aumento importante de la abundancia y riqueza de especies en las comunidades de aves, cuando las franjas de bosque ribereño aumentaron sus anchos, entre $41 \mathrm{~m}$ y $125 \mathrm{~m}$.

Croonquist y Brooks (1993) comentan que en áreas impactadas, de los Estados Unidos para poder conservar las poblaciones reproductivamente viables y las especies que son sensibles a las alteraciones ambientales, es necesario establecer franjas mayores a $25 \mathrm{~m}$. En un censo de 117 franjas ribereñas, cuyos ámbitos oscilaron ente 25 y $800 \mathrm{~m}$ de ancho, Keller et al. (1993) en estudios realizados en Estados Unidos, concluyeron que el incremento en el número de aves es considerable en franjas que van desde los 25 a los $100 \mathrm{~m}$. La información obtenida en este estudio, en bosques tropicales, sugiere que las franjas ribereñas de al menos $50 \mathrm{~m}$ son las que más aportan a la conservación de las poblaciones de aves en la cuenca analizada.

Gremios alimenticios de las comunidades de aves encontrados en los diferentes anchos de franjas de bosques ribereños: Las poblaciones de aves deben asegurar la optimización de la actividad de forrajeo para obtener la máxima cantidad y calidad de alimento con el menor esfuerzo posible (Navarro y Benítez 1995). Una de las maneras de asegurar que exista alimento suficiente es tener habitas disponibles, como es el caso de los bosques ribereños en el paisaje agrícola fragmentado, donde las aves pueden encontrar alimento, y materiales para hacer sus nidos. Además, estos hábitats sirven de refugio para reproducirse y ocultarse de los depredadores. La otra manera de hacerlo es especializarse en un tipo de alimento que sea lo suficientemente abundante y nutritivo para asegurar la supervivencia de la especie, evitando además la competencia con otras especies (Navarro y Benítez. 1995).

El número promedio de individuos (11.42) y especies (6.25) del grupo alimenticio insectívoros y el número promedio de individuos (1.67) y especies (1.25) del grupo alimenticio de nectarívoros fueron más altos en los bosques con franjas ribereñas mayores que $50 \mathrm{~m}$, en comparación con anchos de franjas ribereñas menores que $50 \mathrm{~m}$ (Cuadro 5). La riqueza y abundancia del grupo de insectívoros encontrados en bosques ribereños con franjas mayores, pudo deberse a que en estos ecosistemas, al contar con más área y condiciones climáticas más húmedas que las áreas adyacentes, los insectos son muy abundantes la mayor parte del año; los mismos que son fuentes importantes de nutrientes ricos en proteínas y carbohidratos para las aves (Verea et al. 2000).

En franjas ribereñas mayores a $50 \mathrm{~m}$ también se encontró mayor número de especies e individuos nectarívoros. Esto pudo deberse 
a que estas especies están adaptadas para alimentarse principalmente del néctar de las flores, que es una fuente de alimento rico en energía. En los bosques con franjas ribereñas amplias, la humedad del suelo es mayor, lo que favorece la floración de muchas especies de plantas. Este recurso se presenta también muchas veces, de manera estacional, en los bosques continuos. Los ecosistemas ribereños pueden ofrecer diversidad de flores en diferentes épocas y periodos de tiempo. Terborgh et al. (1990) señalan a las especies de Trochilidae como consumidores oportunistas de recursos estaciónales, para lo cual pueden volar grandes distancias en busca de alimento. Como la floración de las plantas es estacional en los bosques continuos, es posible que algunas de estas especies se muevan entre los bosques ribereños y continuos durante la estación, aprovechando la oferta de flores.

El diámetro de los árboles también estuvo correlacionado con la presencia de especies de nectarívoros; las franjas ribereñas con árboles de mayor diámetro pueden suministrar más alimento a los diferentes grupos alimenticios, debido a que son especies más maduras con una alta productividad de frutos, flores y semillas. La disponibilidad de flores en los ambientes secos es muy marcada, la cercanía de un bosque ribereño donde abundan plantas con flores, unido a la movilidad de las especies de nectarívoros debió favorecer la presencia de este grupo en las franjas de más de $50 \mathrm{~m}$ (Terborgh et al. 1990 citado por Verea et al. 2000).

Los gremios alimenticios de granívoros y carnívoros, no presentaron diferencias ni en el número de individuos ni en el número de especies entre los diferentes anchos de franjas ribereñas. Los mayores valores en estos gremios alimenticios fueron encontrados en los anchos de franja menores de $10 \mathrm{~m}$ (Cuadro 5). Este resultado sugiere que las especies de estos gremios son más generalistas en su alimentación. En el caso las especies de granívoros, Orians (1969) reporta que no dependen tanto del bosque, ya que como los bosques tropicales producen pocas semillas secas, estas especies están restringidas a la vegetación sucesional, creada en los alrededores de las franjas ribereñas, producto de la deforestación, incendios, agricultura y ganadería (Verea y Solórzano 1998).

Dependencia de las poblaciones de aves a los bosques respecto al ancho de franjas ribereñas: De la clasificación de las especies por dependencia al bosque se obtuvo que el $48 \%$ de las especies son independientes de los bosques, el 39\% son dependientes y el 13\% son especies obligadas (totalmente dependientes del bosque). Para todos los grados de dependencia de las aves, se encontraron mayores promedios de especies en las franjas ribereñas mayores que $50 \mathrm{~m}$ en comparación a las franjas con anchos menores que $50 \mathrm{~m}$ : obligadas (4.7), dependientes (7.4) e independientes (14.4), indicando la importancia de las franjas anchas como nichos de alimentación y refugio.

Stiles (1985) indica que las especies obligadas requieren por lo menos de un $50 \%$ de cobertura de bosque, con áreas interconectadas, para su supervivencia y reproducción. Estas especies, si bien son dependientes de bosques considerablemente fragmentados, con una cobertura no menor al 50\% y algunos árboles remanentes (Stiles 1985), pueden forrajear en franjas más angostas. En bosques con franjas de mayor tamaño, la cantidad, variedad de alimento y la disponibilidad de nichos para la reproducción puede determinar la presencia significativa de estas especies.

Las especies dependientes, al igual que las especies independientes, no requieren grandes extensiones de bosques para su supervivencia y se pueden encontrar en cercas vivas o bosques secundarios. Sin embargo, al ser especies muy versátiles, se encuentran en los ecosistemas que brindan mejores ventajas alimentarías y de hábitat, como es el caso de franjas ribereñas mayores de $50 \mathrm{~m}$, que ofrecen mayor variedad de nichos para la reproducción y alimentación (Stiles 1985).

Al incrementarse el ancho de las franjas ribereñas se incrementó también la cantidad de individuos y especies de aves. Esto sugiere que para la conservación de aves migratorias y residentes, en agro-paisajes como la microcuenca del río Sesesmiles, se requiere mantener 
bosques ribereños con franjas de $50 \mathrm{~m}$ de ancho como mínimo. Se encontró mayor número de especies de nectarívoros e insectívoros en las categorías de anchos de franjas ribereñas mayores, lo que sugiere la importancia de mantener estos ecosistemas para la alimentación y reproducción de estos gremios alimenticios. La Ley Forestal de Honduras, en el artículo 64 declara que se deben conservar franjas ribereñas de $150 \mathrm{~m}$ en cada uno de los márgenes de los cauces, sin embargo, actualmente no se respeta el marco legal y se está discutiendo un proyecto de ley, tendiente a respetar solo $30 \mathrm{~m}$ de bosques ribereños en los márgenes de los ríos, lo cual, podría tener impactos negativos en la conservación de las poblaciones de aves y la biodiversidad en general del País.

Los ecosistemas ribereños son importantes no sólo para las especies que se han reportado como propias de los ecosistemas ribereños (Catharus aurantiirostris y Laterallus ruber), sino que también tienen un nivel alto de importancia para las especies generalistas como Turdus grayi, Dives dives y Saltator atriceps en cuanto a habitas, debido a que en los ecosistemas ribereños estas especies pueden encontrar una fuente de alimento y habitas para suplir sus necesidades de supervivencia. El aporte de las franjas ribereñas a la conservación de la biodiversidad de las comunidades de aves, según los resultados de este estudio, fueron favorables.

Dentro de los planes de manejo integrado de cuencas hidrográficas, las instituciones encargadas deberían priorizar la conservación de los bosques ribereños, como un componente estratégico para la gestión ambiental, valorando el aporte de los ecosistemas ribereños a la conservación de la biodiversidad, en paisajes alterados por la agricultura y ganadería.

\section{AGRADECIMIENTOS}

A los pobladores de la microcuenca del río Sesesmiles, personal del programa FOCUENCAS II en Honduras, Municipalidad de Copán Ruinas, por su apoyo técnico y logístico, y los amigos y profesores de CATIE. Este trabajo fue financiado por el Programa FOCUENCAS II, en Honduras.

\section{RESUMEN}

El estudió se realizó en la microcuenca del río Sesesmiles, Departamento de Copán, Honduras. El propósito fue explorar el efecto del ancho de franja de los bosques ribereños sobre la riqueza y abundancia de especies de aves que visitan estos bosques. Se encontraron 1294 aves pertenecientes a 145 especies en bosques ribereños. La diversidad de aves estuvo ligada al ancho de franja de los bosques ribereños: se encontró mayor número de especies e individuos, en anchos iguales o mayores a $50 \mathrm{~m}$. Las especies pertenecientes al grupo alimenticio de los insectívoros y nectarívoros fueron las más abundantes en las franjas ribereñas. Todas las especies de aves identificadas dependen de los bosques ribereños y responden al ancho del bosque; aún las especies más generalistas tienen algún grado de dependencia a las franjas ribereñas más anchas. Para conservar las poblaciones de aves en los paisajes agrícolas fragmentados es de suma importancia mantener franjas ribereñas que sean mayores a $50 \mathrm{~m}$ de ancho y que brinden suficientes habitas y recursos.

Palabras clave: diversidad de aves, dependencia del bosque, bosques ribereños, grupos alimentarios.

\section{REFERENCIAS}

Croonquist, M. \& R. Brooks. 1993. Effects of habitat disturbance on bird communities in riparian corridors. JS.W.C.A 48: 65-70.

Finegan, B., J. Hayes, D. Delgado \& S. Gretzinger. 2004. Monitoreo ecológico del manejo forestal en el trópico húmedo: una guía para operadores forestales \& certificadores con énfasis en Bosques de Alto Valor para la Conservación. WWF Centroamérica, PROARCA, CATIE y OSU, Turrialba, Cartago, Costa Rica.

Howell, S. \& S. Webbs. 1995. A Guide to the Birds of Mexico and Northern Central America. Oxford, Oxford, Inglaterra.

Keller, C., C. Robbins \& J. Hatfield. 1993. Avian communities in riparian forest of different widths in Maryland and Delaware. Wetlands 13: 137-44.

Klimas, C.V., O. Martin \& J. Teaford. 1981. Impacts of flooding regime modification on wildlife habitats of bottomland hardwood forest in the lower Mississippi 
Valley. Tech. Rep. E1-81-13. US Army Engineers Water-ways Exp. Station, Vicksburg, Missouri, EEUU.

Kauffman, J. \& W. Kruger. 1984. Livestock impacts on riparian ecosystems and streamside management implications: A review. J. Range Manage. 37: 430-438.

Lock, P. \& R. Naiman. 1998. Effects of stream size on bird community structure in coastal temperate forests of the Pacific Northwest, U.S.A. J. Biogeography 25: 773-782.

McLean, A., H. Nicholson \& A. Van Ryswyk. 1963. Growth productivity and chemical composition of a subalpine meadow in interior British Columbia. J. Range Management 16: 235-240.

Navarro, A. \& H. Benítez. 1995. Fondo de cultura económica. México DF, México (disponible también en Internet: www.omega.ilce.edu.mx:3000/sites/ciencia/ volumen3/ciencia3/138/htm/dominio.htm)

Nores, M., M. Cerana \& D. Serra. 2005. Dispersal of forest birds and trees along the Uruguay River in South America. Diversity. Distrib. 11: 13

Orians, G. 1969. The number of bird species in some tropical forests. Ecology 50: 783-801.

Paulsen, H.A.Jr. 1969. Forage value on a mountain grassland aspen range in western Colorado. J. Range Management. 22:102-107.

Ralph, C., G. Geupel, P. Pyle, T. Martin, D. DeSante \& B. Milá. 1996. Manual de métodos de campo para el monitoreo de aves terrestres. Pacific Southwest Research Station, Forest Service, U.S. Department of Agriculture, Washington, DC.

Ralph, C. 1995. Interannual differences in detections of Marbled Murrelets in some inland California stands. USDA Forest Service. PSW-GTR-152: 135-138.

Shirley, S. \& J. Smith. 2005. Bird community structure across riparian buffer strips of varying widths in a coastal temperate forest. Biol. Conserv. 125: 475-489.

Skagen, S., C. Melcher, W. Howe \& F. Knopf. 1998. Comparative use of riparian corridors and oases by migrating birds in southeast Arizona. Conserv. Biol. 12: $896-909$
Skovlin, J. 1967. Fluctuations in forage quality on summer rage in the Blue Mountains. USDA Forest Service. PNW-Res, United States Department of Agriculture, EEUU.

Smith, L. \& R. Johnstone. E. 1977. Status of the Purplecrowned Wren (Malurus coronatus) and Buffsided robin (Poecilodryas superciliosa) in Western Australia. Western Australia. Naturalist 13:185-88.

Spackman, S. \& J. Hughes. 1995. Assessment of minimum stream corridor width for biological conservation: species richness and distribution along mid-order streams in Vermont, EEUU. Biol. Conserv. 71: 325-332.

Stauffer, D. \& L. Best. 1980. Habitat selection by birds of riparian communities: evaluating effects of habitat alterations. J. Wildlife. Management. 44: 15.

STILES FG (1985) Conservation of forest birds in Costa Rica: problems and perspectives, pp. 141-168. In Diamond AW \& TE Lovejoy (eds.). Conservation of tropical forest birds. International Council for Bird Preservation, Cambridge, Reino Unido.

Stiles, F. \& A. Skutch. 1989. A guide to the birds of Costa Rica. Cornell University. Ithaca, Nueva York. EEUU.

Terborgh, J., S. Robinson, T. Parker, C. Munn \& N. Pierpont. 1990. Structure and organization of an Amazonian forest bird community. Ecol. Mon. 60: 213-238.

Treviño, G., C. Cavazos \& O. Aguirre. 2001. Distribución y estructura de los bosques de galería en dos ríos del centro sur de Nuevo León. Madera y Bosques 7: 13-25.

Villón, M. 2002. Hidrología. Instituto Tecnológico de Costa Rica. Escuela de Ingeniería Agrícola. Comité Regional de Recursos Hidráulicos. Cartago, Costa Rica.

Villarreal, H., M. Álvarez, F. Córdoba, G. Escobar, G. Fagua, F Gast, H. Mendoza, M. Ospina \& A. Umaña. 2004. Manual de métodos para el desarrollo de inventarios de biodiversidad. Programa de inventarios de Biodiversidad. Instituto de Investigación de Recursos Biológicos Alexander von Humboldt. Bogotá, Colombia.

Verea, C., A. Badillo \& A. Solórzano. 2000. Variación en la composición de las comunidades de aves de sotobosque de dos bosques en el norte de Venezuela. Ornitol. Neotrop. 11: 65-79. 
Verea, C. \& Solórzano, A. 1998. La avifauna del sotobosque de una selva decidua tropical en Venezuela. Ornitol. Neotrop. 9: 161-176.

Woinarski, J., C. Brock, M. Armstrong, D. Hempel, D. Cheal \& K. Brennan. 2000. Bird distribution in riparian vegetation of an Australian tropical savanna: a broad-scale survey and analysis of distributional data base. J. Biogeography 27: 843-868.

\section{OTRAS REFERENCIAS}

InfoStat, 2004. InfoStat, versión 2004 (programa estadístico). Grupo InfoStat, FCA, Universidad Nacional de Córdoba, Córdoba, Argentina.

Navarro, A. \& H. Benítez. 1995. Sin título. Fondo de Cultura Económica. México D.F. (www.omega.ilce. edu.mx:3000/sites/ciencia/volumen3/ciencia3/138/ htm/dominio.htm consultada 20 octubre 2005). 
